\title{
Light and heavy mesons in a soft-wall holographic approach
}

\author{
Tanja Branz ${ }^{1}$, Thomas Gutsche ${ }^{1}$, Valery E. Lyubovitskij ${ }^{*}$, Ivan Schmidt ${ }^{2}$, Alfredo Vega ${ }^{2}$ \\ 1 Institut für Theoretische Physik, Universität Tübingen, \\ Kepler Center for Astro and Particle Physics, \\ Auf der Morgenstelle 14, D-72076 Tübingen, Germany \\ 2 Departamento de Física y Centro Científico Tecnológico de Valparaíso (CCTVal), \\ Universidad Técnica Federico Santa María, \\ Casilla 110-V, Valparaíso, Chile
}

(Dated: September 27, 2018)

We study the mass spectrum and decay constants of light and heavy mesons in a soft-wall holographic approach, using the correspondence of string theory in Anti-de Sitter space and conformal field theory in physical space-time.

PACS numbers: 13.20.Gd, 13.20.He, 14.40.Ak, 14.40.Lb, 14.40.Nd

Keywords: light and heavy mesons, holographic model, mass spectrum, decay constants

* On leave of absence from Department of Physics, Tomsk State University, 634050 Tomsk, Russia 


\section{INTRODUCTION}

In a series of papers [1]-8] Brodsky and de Téramond developed a semiclassical approximation to QCD - lightfront holography (LFH)-, an approach based on the correspondence of string theory in Anti-de Sitter (AdS) space and conformal field theory (CFT) in physical space-time [9, 10]. Light-front holography [1]-[8] is one of the exciting features of the AdS/CFT correspondence. The LFH approach is a covariant and analytic model for hadron structure with confinement at large and conformal behavior at short distances. It is analogous to the Schrödinger theory for atomic physics. It provides a precise mapping of the string modes $\Phi(z)$ in the AdS fifth dimension $z$ to the hadron light-front wave functions (LFWF) in physical space-time, in terms of the light-front impact variable $\xi$, which measures the separation of the quark and gluonic constituents inside a hadron. Therefore, different values of the holographic variable $z$ correspond to different scales at which the hadron is examined. The mapping was obtained by matching certain matrix elements (e.g. electromagnetic pion form factor, the energy-momentum tensor) in the two approaches - string theory in AdS and light-front theory in Minkowski space-time.

In order to break conformal invariance and incorporate confinement in the infrared (IR) region, two alternative AdS/QCD backgrounds have been suggested in the literature: the "hard-wall" approach [11]-[19], based on introducing an IR brane cutoff in the fifth dimension, and the "soft-wall" approach [2], 20]-37], based on using a soft cutoff by introducing a background dilaton field in the AdS space or using a warp factor in the metric.

Both approaches have certain advantages. One of the problems of the "hard-wall" scenario is a linear dependence of hadron masses with $M \propto L$ for higher values of the orbital momentum $L$ instead of the quadratic behavior $M^{2} \propto L$ (known as Regge trajectory). In fact, the "soft-model" was initiated in order to solve the problem of the hadronic mass spectrum. In Refs. [2] and [20]-37] the "soft-model" has been applied to different aspects of hadron properties, including the hadron and glueball mass spectrum, the heavy quark potential, form factors, deep inelastic scattering, etc. Notice that in the LFH approach [1]-[8] both scenarios for AdS/QCD backgrounds ("hard-wall" and "soft-wall") are used in order to map the string modes to the LFWF restricting to the case of massless quarks. A generalization of the LFWF for massive quarks has been suggested by Brodsky and de Téramond, including quark masses explicitly in the LF kinetic energy $\sum_{i}\left(\mathbf{k}_{\perp i}^{2}+m_{i}^{2}\right) / x_{i}$ (see details in Ref. [3]). The LFH approach has been successfully applied to the description of the mass spectrum of meson and baryons (reproducing the Regge trajectories), the pion leptonic constant, the electromagnetic form factors of pion and nucleons, etc. [1]-[8]. In Refs. [28, 29] an alternative softwall holographic model has been developed, which provides an extension to hadrons with an arbitrary number of constituents. In Ref. 31 meson wave functions derived in these two approaches were discussed in the case of massive quarks.

Notice that the LFH approach developed in Ref. [1]-[8] uses the so-called "negative" dilaton field profile $\left(e^{-\phi(z)}\right.$ with $\left.\phi(z)=-\kappa^{2} z^{2}\right)$, which was necessary to produce a massless pion and the behavior of the gravitational potential. In this paper we use the soft-wall approach with a "positive" dilaton field profile $\phi(z)=\kappa^{2} z^{2}$, as suggested originally in Ref. [20]. In the context of the original soft-wall model [20]-[37], the positive sign in the dilaton profile is important to reproduce the correct behavior of Regge trajectories for higher spin states. In fact, as stressed in a recent paper [37], the two different signs of the dilaton field profile are related to two different ways of introducing higher spin fields. On the other hand, as was shown in Refs. [17, 20, 26, 30], the pion appears massless in soft-wall models with a "positive" dilaton. Moreover, one of the outstanding features of the LFH approach [1]-[8] is the LF mapping - matching of QCD LF dynamics with the corresponding string dynamics in AdS space. This was, for example, done in the case of the pion electromagnetic form factor (see details in [2]). In particular, in the calculation of the pion form factor at large values of the Euclidean momentum squared it was shown that the dilaton should have a positive sign.

In this manuscript we show that the use of the positive dilaton in the LFH approach [1]-[8] is possible if we modify the mass term of the $\mathrm{AdS}_{d+1}$ action. The proposed approach is applied to the study of the mass spectrum and decay properties of light and heavy mesons. In the case of the mass spectrum we include color Coulomb and hyperfinesplitting corrections. The paper is structured as follows. First, in Sec. II, we briefly discuss the basic notions of the approach. In Sec. III, we consider the mass spectrum and decay properties of light and heavy mesons. Finally, in Sec. IV, we summarize our results.

\section{BASIC APPROACH}

\section{A. AdS action and Schrödinger equation of motion for mesons}

Our starting point is the action in $\mathrm{AdS}_{d+1}$ spacetime for a spin- $J$ field $\Phi_{J}=\Phi_{M_{1} \cdots M_{J}}(x, z)$ - a symmetric, traceless tensor used in the LFH approach [1]-8], where we perform two modifications: 1) we use a positive dilaton profile $\left.\phi(z)=\kappa^{2} z^{2} ; 2\right)$ we include a nontrivial $z$-dependence of the mass term coefficient $\mu_{J}^{2} \rightarrow \mu_{J}^{2}(z)$ due to the interaction 
of the dilaton field with the matter field:

$$
S_{\Phi}=\frac{(-1)^{J}}{2} \int d^{d} x d z \sqrt{g} e^{-\phi(z)}\left(\partial_{N} \Phi_{J} \partial^{N} \Phi^{J}-\mu_{J}^{2}(z) \Phi_{J} \Phi^{J}\right),
$$

where $\mu_{J}^{2}(z)=\mu_{J}^{2}+g_{J} \phi(z)$ is the "dressed" mass due to the interaction of the dilaton with $\Phi_{J}$. Note that a similar modification of the mass term of the string mode dual to the spinor field describing nucleons has been done in the context of the soft-wall model in Ref. [34]. The coupling constant $g_{J}$ will be fixed later in order to get a massless pion. The metric is defined as

$$
d s^{2}=\left(\frac{R}{z}\right)^{2}\left(\eta_{\mu \nu} d x^{\mu} d x^{\nu}-d z^{2}\right), \quad \eta_{\mu \nu}=\operatorname{diag}(1,-1, \ldots,-1) .
$$

where $R$ is the AdS radius, $g=\left|\operatorname{det} g_{M N}\right|=(R / z)^{2(d+1)}$, and $g_{M N}$ is the metric tensor of $d+1$ space.

Next we restrict to the axial gauge $\Phi_{z \ldots}(x, z)=0$. We consider the string modes dual to hadrons with total angular momentum $J$, four-momentum $P$ and propagating in AdS space along the Poincaré coordinates:

$$
\Phi_{\nu_{1} \cdots \nu_{J}}(x, z)=\sum_{n} \varphi_{n J}(z) \int \frac{d^{d} P}{(2 \pi)^{d}} e^{-i P x} \epsilon_{\nu_{1} \cdots \nu_{J}}^{n}(P)
$$

where $\nu_{1} \cdots \nu_{J}$ are the Poincaré indices, $n$ is the radial quantum number and $\epsilon_{\nu_{1} \cdots \nu_{J}}^{n}(P)$ is the polarization tensor.

Performing the substitution

$$
\varphi_{n J}(z)=e^{\frac{\phi(z)}{2}}\left(\frac{R}{z}\right)^{J-\frac{d-1}{2}} \Phi_{n J}(z)
$$

one can derive the Schrödinger-type equation of motion $(\mathrm{EOM})$ for $\Phi_{n J}(z)$ :

$$
\left[-\frac{d^{2}}{d z^{2}}+U_{J}(z)\right] \Phi_{n J}(z)=M_{n J}^{2} \Phi_{n J}(z)
$$

where $U_{J}(z)$ is the effective potential given by

$$
U_{J}(z)=\kappa^{4} z^{2}+\frac{4 a_{J}^{2}-1}{4 z^{2}}+2 \kappa^{2}\left(b_{J}-1\right)
$$

with

$$
a_{J}=\frac{1}{2} \sqrt{(d-2 J)^{2}+4\left(\mu_{J} R\right)^{2}}, \quad b_{J}=\frac{1}{2}\left(g_{J} R^{2}+d-2 J\right) .
$$

Analytical solutions of Eq. (5) - eigenfunctions and eigenvalues are:

$$
\Phi_{n J}(z)=\sqrt{\frac{2 n !}{\left(n+a_{J}\right) !}} \kappa^{1+a_{J}} z^{1 / 2+a_{J}} e^{-\kappa^{2} z^{2} / 2} L_{n}^{a_{J}}\left(\kappa^{2} z^{2}\right)
$$

and

$$
M_{n J}^{2}=4 \kappa^{2}\left(n+\frac{a_{J}+b_{J}}{2}\right)
$$

where

$$
L_{n}^{m}(x)=\frac{x^{-m} e^{x}}{n !} \frac{d^{n}}{d x^{n}}\left(e^{-x} x^{m+n}\right)
$$

are the generalized Laguerre polynomial.

Restricting to $d=4$ with $\left(\mu_{J} R\right)^{2}=L^{2}-(2-J)^{2}[1]$ - [8] we fix the value $g_{J} R^{2}=4(J-1)$ in order to get a massless pion. Therefore, in the case $d=4$ we get $a_{J}=L$ and $b_{J}=J$, and the solutions of the Schrödinger-type equation read as:

$$
\Phi_{n J}(z)=\sqrt{\frac{2 n !}{(n+L) !}} \kappa^{1+L} z^{1 / 2+L} e^{-\kappa^{2} z^{2} / 2} L_{n}^{L}\left(\kappa^{2} z^{2}\right)
$$


and

$$
M_{n J}^{2}=4 \kappa^{2}\left(n+\frac{L+J}{2}\right) .
$$

Here we do not divide the total angular momentum $J$ into the quantum numbers of the quark-antiquark pair orbital angular momentum $L$ and internal spin $S$. Such a model ansatz was done in Refs. [1] - [8] . Because of $J=L$ or $J=L \pm 1$ the present soft-wall model generates linear Regge trajectories in both quantum numbers $n$ and $J$ (or $L$ ): $M_{n J}^{2} \sim n+J$. Note that the string modes dual to the pseudoscalar $J^{P C}=0^{-+}$and scalar $J^{P C}=0^{++}$ mesons, and correspondingly the vector $J^{P C}=1^{--}$and axial $J^{P C}=1^{++}$mesons, are different from each other (mass spectrum and wave functions) via the mass parameter of the string mode $\left(\mu_{J} R\right)^{2}$, depending explicitly on the orbital momentum $L$. Inclusion of chiral symmetry breaking effects in the AdS action and their impact on the hadron properties will be analyzed in the future.

\section{B. AdS and light-front QCD correspondence}

The string mode $\Phi_{n J}(z)$ can be directly mapped to the LFWF due to the correspondence of AdS and light-front amplitudes. In particular, considering the case of two partons $q_{1}$ and $\bar{q}_{2}$ the holographic coordinate $z$ is related to the impact variable $\zeta$ in the $\mathrm{LF}$ formalism as

$$
z \rightarrow \zeta, \quad \zeta^{2}=\mathbf{b}_{\perp}^{2} x(1-x)
$$

where $\mathbf{b}_{\perp}$ is the transverse coordinate and Fourier conjugate to the transverse momentum $\mathbf{k}_{\perp}$. In the massless case we obtain a relation between the AdS modes and the meson LFWF $\widetilde{\psi}_{q_{1} \bar{q}_{2}}(x, \zeta)[31$ :

$$
\left|\widetilde{\psi}_{q_{1} \bar{q}_{2}}(x, \zeta)\right|^{2}=P_{q_{1} \bar{q}_{2}} x(1-x) f^{2}(x) \frac{\left|\Phi_{n J}(\zeta)\right|^{2}}{2 \pi \zeta}
$$

where $P_{q_{1} \bar{q}_{2}}$ is the the probability of finding the valence Fock state $\left|q_{1} \bar{q}_{2}\right\rangle$ in the meson $M$ :

$$
P_{q_{1} \bar{q}_{2}}=\int_{0}^{1} d x \int d^{2} \mathbf{b}_{\perp}\left|\widetilde{\psi}_{q_{1} \bar{q}_{2}}\left(x, \mathbf{b}_{\perp}\right)\right|^{2} \leq 1 .
$$

In the following we restrict to the case of $P_{q_{1} \bar{q}_{2}}=1$ and only for the pion we consider $P_{q_{1} \bar{q}_{2}}<1$ (see discussion in Ref. [31]). Here $f(x)$ is the longitudinal mode which is normalized as $\int_{0}^{1} d x f^{2}(x)=1$. In our case $f(x) \equiv 1$. Then the expressions for the meson LFWFs read:

$$
\widetilde{\psi}_{q_{1} \bar{q}_{2}}\left(x, \mathbf{b}_{\perp}\right)=\sqrt{\frac{2 n !}{(n+L) !}} \frac{\kappa^{1+L}}{\sqrt{\pi}}\left|\mathbf{b}_{\perp}\right|^{L}[x(1-x)]^{\frac{1+L}{2}} e^{-\frac{1}{2} \kappa^{2} x(1-x) \mathbf{b}_{\perp}^{2}} L_{n}^{L}\left(\kappa^{2} \mathbf{b}_{\perp}^{2} x(1-x)\right) .
$$

The meson LFWF (16) does not consider massive quarks. The inclusion of finite quark masses has been considered by us previously in [31]. In particular, the quark masses in the meson LFWF have been included following a prescription suggested by Brodsky and de Téramond [3]. Here we illustrate this procedure for the ground state LFWFs. First one should take the Fourier transform of (16)

$$
\psi_{q_{1} \bar{q}_{2}}\left(x, \mathbf{k}_{\perp}\right)=\frac{4 \pi}{\kappa \sqrt{x(1-x)}} e^{-\frac{\mathbf{k}_{\perp}^{2}}{2 \kappa^{2} x(1-x)}} .
$$

In a second step the quark masses are introduced by extending the kinetic energy of massless quarks with $K_{0}=\frac{\mathbf{k}_{\perp}^{2}}{x(1-x)}$ to the case of massive quarks:

$$
K_{0} \rightarrow K=K_{0}+m_{12}^{2}, \quad m_{12}^{2}=\frac{m_{1}^{2}}{x}+\frac{m_{2}^{2}}{1-x} .
$$

Note that the change proposed in (18) is equivalent to the following change of the kinetic term in the Schrödinger EOM:

$$
-\frac{d^{2}}{d \zeta^{2}} \rightarrow-\frac{d^{2}}{d \zeta^{2}}+m_{12}^{2}
$$


Finally we obtain

$$
\psi_{q_{1} \bar{q}_{2}}\left(x, \mathbf{k}_{\perp}\right)=\frac{4 \pi N}{\kappa \sqrt{x(1-x)}} e^{-\frac{\mathbf{k}_{\perp}^{2}}{2 \kappa^{2} x(1-x)}-\frac{m_{12}^{2}}{2 \kappa^{2}}} .
$$

As was suggested in [5], the quark mass term in the exponential of Eq. (20) can be absorbed in the longitudinal mode for massive quarks

$$
f\left(x, m_{1}, m_{2}\right) \equiv N f(x) e^{-\frac{m_{12}^{2}}{2 \kappa^{2}}}
$$

where $N$ is the normalization constant fixed from

$$
1=\int_{0}^{1} d x f^{2}\left(x, m_{1}, m_{2}\right)
$$

Hence, the meson LFWFs with massive quarks can be written down as a product of transverse $\Phi(\zeta)$, longitudinal $f\left(x, m_{1}, m_{2}\right)$ and angular $e^{i m \phi}$ modes [5]:

$$
\widetilde{\psi}_{q_{1} \bar{q}_{2}}\left(x, \zeta, m_{1}, m_{2}\right)=\frac{\Phi_{n J}(\zeta)}{\sqrt{2 \pi \zeta}} f\left(x, m_{1}, m_{2}\right) e^{i m \phi} \sqrt{x(1-x)},
$$

where $m=0, \pm 1, \pm 2, \cdots, \pm L$ is the magnetic quantum number. One should stress that the way in which massive quarks are introduced is not unique. In particular, the dimensional parameter entering in the longitudinal mode $f\left(x, m_{1}, m_{2}\right)$ should not necessarily be identified with the parameter $\kappa$ characterizing the dilaton field. Later, in the analysis of the mass spectrum and the decay constants of heavy-light mesons, we will show that the dilaton parameter $\kappa$ should scale as $\mathcal{O}(1)$ in the $1 / m_{Q}$ expansion, where $m_{Q}$ is the heavy quark mass, while the dimensional parameter in the longitudinal mode should scale as $\mathcal{O}\left(m_{Q}^{1 / 2}\right)$. In the case of heavy quarkonia the dimensional parameter in the longitudinal mode should scale as $\mathcal{O}\left(m_{Q}\right)$. Hence, for the longitudinal mode we will use the functional form

$$
f\left(x, m_{1}, m_{2}\right) \equiv N f(x) e^{-\frac{m_{12}^{2}}{2 \lambda_{12}^{2}}},
$$

containing quark masses and an additional scale parameter $\lambda_{12}$.

The meson mass spectrum in the case of massive quarks is given by [5]:

$$
M_{n J}^{2}=\int_{0}^{\infty} d \zeta \Phi_{n J}(\zeta)\left(-\frac{d^{2}}{d \zeta^{2}}-\frac{1-4 L^{2}}{4 \zeta^{2}}+\kappa^{4} \zeta^{2}+2 \kappa^{2}(J-1)\right) \Phi_{n J}(\zeta)+\int_{0}^{1} d x\left(\frac{m_{1}^{2}}{x}+\frac{m_{2}^{2}}{1-x}\right) f^{2}\left(x, m_{1}, m_{2}\right)
$$

This means that for massive quarks the hadron masses are shifted due to the last term in the r.h.s. of Eq. (25). One should stress that the potential in Eq. (25) is not complete. It includes confinement forces but does not include in its full context effects of chiral symmetry breaking, which are important for consistency with the infrared structure of QCD (see e.g. the discussion in Refs. [7, 8, 17, 20, 26, 27, 30, 38]). Moreover, it does not contain the one-gluon exchange term, which is sufficient for the description of bottomia hadrons, and also hyperfine-splitting terms. As we stressed before, we intend to include chiral symmetry breaking in the formalism in a forthcoming work.

\section{One-gluon exchange and hyperfine--splitting contributions to the effective meson potential}

We will include the one-gluon exchange and hyperfine-splitting terms phenomenologically by extending the effective potential $U \rightarrow U+U_{\mathrm{C}}+U_{\mathrm{HF}}$, where $U_{\mathrm{C}}$ and $U_{\mathrm{HF}}$ are the contributions of the color Coulomb and hyperfine (HF) splitting potentials.

Note that, as it was stressed in [39, 40], the trajectories of bottomia states deviate from linearity. The reason is that, due to the one-gluon exchange term, there is an additional Coulomb-like interaction between quarks $V(r)=-4 \alpha_{s} / 3 r$, where $\alpha_{s}$ is the strong coupling constant. Its contribution to the mass spectrum $M^{2}$ is negative and proportional to the quark mass squared [39, 40]. Therefore, for light mesons and charmonia states this term can be neglected, while 
this is not the case for the bottomia states. Extending the result of Refs. 39, 40 to the general case of a meson containing constituent quarks with masses $m_{1}$ and $m_{2}$, we get the following expression for the shift of $M^{2}$ due to the color Coulomb potential:

$$
\Delta M_{\mathrm{C}}^{2}=-\frac{64 \alpha_{s}^{2} m_{1} m_{2}}{9(n+L+1)^{2}}
$$

where $\alpha_{s}$ is the QCD coupling, considered as a free parameter. The Coulomb potential, which should be included in the effective meson potential $U(\zeta)$, reads

$$
U_{\mathrm{C}}(\zeta)=-\frac{\sigma}{\zeta}
$$

where the coupling constant $\sigma$ is fixed as

$$
\sigma=\frac{64 \alpha_{s}^{2} m_{1} m_{2}}{9(n+L+1)^{2}}\left\{\int_{0}^{\infty} \frac{d \zeta}{\zeta} \Phi_{n J}^{2}(\zeta)\right\}^{-1}
$$

For the hyperfine-splitting potential $U_{\mathrm{HF}}(\zeta)$ one can use an effective operator containing a free parameter $v$ (softening the original $\delta$-functional form of the HF-potential) having dimension $M^{3}$ [see details in Refs. [41, 42]]:

$$
U_{\mathrm{HF}}(\zeta)=\frac{32 \pi \alpha_{s}}{9} \frac{\boldsymbol{\sigma}_{\mathbf{1}} \boldsymbol{\sigma}_{\mathbf{2}}}{\mu_{12}} v
$$

where $\boldsymbol{\sigma}_{\mathbf{1}}$ and $\boldsymbol{\sigma}_{\mathbf{2}}$ are the spin operators of the quarks; $\mu_{12}=2 m_{1} m_{2} /\left(m_{1}+m_{2}\right)$. Projecting the operator $\boldsymbol{\sigma}_{\mathbf{1}} \boldsymbol{\sigma}_{\mathbf{2}}$ between meson states with $S=0,1$ gives

$$
\beta_{S}=\left\langle M_{S}\left|\sigma_{1} \sigma_{\mathbf{2}}\right| M_{S}\right\rangle=\left\{\begin{array}{cc}
-3, & S=0 \\
1, & S=1
\end{array} .\right.
$$

Therefore, the mass shift due to the hyperfine-splitting potential is

$$
\Delta M_{\mathrm{HF}}^{2}=\frac{32 \pi \alpha_{s}}{9} \frac{\beta_{S} v}{\mu_{12}} .
$$

Finally, the master formula for meson masses including confinement, color Coulomb and hyperfine-splitting effects reads:

$$
M_{n J}^{2}=4 \kappa^{2}\left(n+\frac{L+J}{2}\right)+\int_{0}^{1} d x\left(\frac{m_{1}^{2}}{x}+\frac{m_{2}^{2}}{1-x}\right) f^{2}\left(x, m_{1}, m_{2}\right)-\frac{64 \alpha_{s}^{2} m_{1} m_{2}}{9(n+L+1)^{2}}+\frac{32 \pi \alpha_{s}}{9} \frac{\beta_{S} v}{\mu_{12}} .
$$

There are two comments that should be made with respect to further modifications of the potential $U$. First, as suggested in Ref. [35] the dilaton scale parameter can be different for distinct types of mesons - light and heavy ones. In particular, we observe that the use of a larger value of $\kappa$ for heavy mesons helps to improve the description of the mass spectrum and the leptonic decay constants. Second, in Ref. [36] it was suggested to add a constant term $c^{2}$ to the effective potential which is independent on the parameter $\kappa$ and controls the masses of the ground states. In our formalism such a constant term in the effective potential can be e.g. generated by an additional shift of the "dressed" mass term $\mu_{J}^{2}(z) \rightarrow \mu_{J}^{2}(z)+c^{2} z^{2} / R^{2}$, which leads to the following modification of the mass spectrum: $M_{n J}^{2} \rightarrow M_{n J}^{2}+c^{2}$. Although both of these modifications can improve the description of meson properties, their appearance in the AdS action is not well justified. Therefore, in the present manuscript we do not consider these options and postpone them for future study.

\section{PROPERTIES OF LIGHT AND HEAVY MESONS}

\section{A. Mass spectrum of light mesons}

In the numerical analysis we restrict ourselves to the isospin limit $m_{u}=m_{d}=m$. We fix the free parameters (constituent quark masses, $\kappa, \lambda_{12}, \alpha_{s}$ and $v$ ) from a fit to the mass spectrum and the decay constants of light and 
heavy mesons. Note that we use a unified value for the dilaton parameter $\kappa$ for all meson states as dictated by the AdS action.

The parameters are fixed to the following values. For the constituent quark masses we have:

$$
m=420 \mathrm{MeV}, \quad m_{s}=570 \mathrm{MeV}, \quad m_{c}=1.6 \mathrm{GeV}, \quad m_{b}=4.8 \mathrm{GeV} .
$$

The unified value of the dilaton parameter is fixed as $\kappa=550 \mathrm{MeV}$ for all mesons. The hyperfine-splitting parameter has the value $v=10^{-4} \mathrm{GeV}^{3}$. The strong coupling $\alpha_{s} \equiv \alpha_{s}\left(\mu_{12}^{2}\right)$ depends on the quark flavor and is consistently calculated using the parametrization of $\alpha_{s}$ with "freezing" [43]:

$$
\alpha_{s}\left(\mu^{2}\right)=\frac{12 \pi}{\left(33-2 N_{f}\right) \ln \frac{\mu^{2}+M_{B}^{2}}{\Lambda^{2}}}
$$

where $N_{f}$ is the number of flavors, $\Lambda$ is the QCD scale parameter and $M_{B}$ is the background mass. Choosing $\Lambda=420 \mathrm{MeV}, M_{B}=854 \mathrm{MeV}$ and using the fixed constituent quark masses from Eq. (33) we obtain the following set of parameters $\alpha_{s}$ :

$$
\begin{aligned}
& \alpha_{s}\left(\mu_{q q}^{2}\right)=0.79, \alpha_{s}\left(\mu_{q s}^{2}\right)=0.77, \alpha_{s}\left(\mu_{s s}^{2}\right)=0.78, \alpha_{s}\left(\mu_{q c}^{2}\right)=0.68, \alpha_{s}\left(\mu_{s c}^{2}\right)=0.67, \\
& \alpha_{s}\left(\mu_{u b}^{2}\right)=0.64, \alpha_{s}\left(\mu_{s b}^{2}\right)=0.61, \alpha_{s}\left(\mu_{c c}^{2}\right)=0.52, \alpha_{s}\left(\mu_{c b}^{2}\right)=0.42, \alpha_{s}\left(\mu_{b b}^{2}\right)=0.33 \text {, }
\end{aligned}
$$

where $q=u, d$. The dimensional parameters $\lambda_{12}$ in the longitudinal wave functions are fitted as:

$$
\begin{aligned}
& \lambda_{q q}=0.63 \mathrm{GeV}, \quad \lambda_{u s}=1.2 \mathrm{GeV}, \quad \lambda_{s s}=1.68 \mathrm{GeV}, \quad \lambda_{q c}=2.5 \mathrm{GeV}, \quad \lambda_{s c}=3.0 \mathrm{GeV} \\
& \lambda_{q b}=3.89 \mathrm{GeV}, \quad \lambda_{s b}=4.18 \mathrm{GeV}, \quad \lambda_{c c}=4.04 \mathrm{GeV}, \quad \lambda_{c b}=4.82 \mathrm{GeV}, \quad \lambda_{b b}=6.77 \mathrm{GeV}
\end{aligned}
$$

Here we also already indicate the values used for the heavy-light and heavy mesons. For the probabilities of the ground state pion and kaon we use the following values: $P_{\pi}=0.6$ and $P_{K}=0.8$, while for other mesons the probabilities are supposed to be equal to 1 .

The predictions of our approach for the light meson spectrum according to the $n^{2 S+1} L_{J}$ classification are given in Table I. For the scalar mesons $f_{0}$ we present results for two limiting cases: with nonstrange flavor content $f_{0}[\bar{n} n]=$ $(\bar{u} u+\bar{d} d) / \sqrt{2}$ and with a strange one $f_{0}[\bar{s} s]=\bar{s} s$.

\section{B. Mass spectrum of heavy-light mesons}

Before we apply our approach to the properties of heavy mesons we would like to check if our LFWFs are consistent with model-independent constraints imposed e.g. by heavy quark symmetry, when the heavy quark mass goes to infinity $m_{Q} \rightarrow \infty$.

The mass spectrum of heavy-light mesons is given by the formula

$$
M_{q Q}^{2}=4 \kappa^{2}\left(n+\frac{L+J}{2}\right)+\int_{0}^{1} d x\left(\frac{m_{q}^{2}}{x}+\frac{m_{Q}^{2}}{1-x}\right) f^{2}\left(x, m_{q}, m_{Q}\right)-\frac{64 \alpha_{s}^{2} m_{q} m_{Q}}{9(n+L+1)^{2}}+\frac{32 \pi \alpha_{s}}{9} \frac{\beta_{S} v}{\mu_{q Q}}
$$

The longitudinal mode for heavy-light mesons is of the form

$$
f\left(x, m_{q}, m_{Q}\right) \equiv N f(x) e^{-\frac{m_{q Q}^{2}}{2 \lambda_{q Q}^{2}}}
$$

where $\lambda_{q Q}$ is the dimensional parameter which scales as $\mathcal{O}\left(m_{Q}^{1 / 2}\right)$. In the following, for convenience, we express $\lambda_{q Q}$ as $\lambda_{q Q}^{2}=m_{q} m_{Q} / r$, where $r$ is a parameter of order $\mathcal{O}(1)$. The scaling of the parameter $\kappa \sim \mathcal{O}(1)$ is fixed by the scaling law of the leptonic constants of heavy-light mesons in the heavy quark limit (see discussion in Sec. IIID). This behavior of $\kappa$ is also consistent with the mass spectroscopy of heavy-light mesons constrained by heavy quark effective theory (HQET) [44]. In particular, the $1 / m_{Q}$ expansion of their masses should be

$$
M_{q Q}=m_{Q}+\bar{\Lambda}+\mathcal{O}\left(1 / m_{Q}\right)
$$


where the scale parameter $\bar{\Lambda}$ is of order $\mathcal{O}(1)$, and the mass splitting of vector and pseudoscalar states $\Delta M_{q Q}=$ $M_{q Q}^{V}-M_{q Q}^{P}$ should be of order $1 / m_{Q}$ :

$$
\Delta M_{q Q}=\frac{2}{M_{q Q}^{V}+M_{q Q}^{P}}\left(\kappa^{2}+\frac{64 \pi \alpha_{s}}{9} \frac{\beta_{S} v}{m_{q}}\right) \sim \frac{1}{m_{Q}} .
$$

The mass splitting $\Delta M_{q Q}$ gets contributions from two sources - confinement and the hyperfine-splitting potential. Both contributions are of order $\mathcal{O}(1)$ in the heavy quark mass expansion. In Appendix $\mathrm{B}$ we evaluate the r.h.s. of Eq. (37) and give an expression for the scale parameter $\bar{\Lambda}$.

Numerical values for the charm and bottom heavy-light mesons with different spin-parity are given in Table II according to the $n^{2 S+1} L_{J}$ classification. The four columns with the results correspond to the variation of $L=0,1,2,3$. Data for the ground states are given in the brackets.

\section{Mass spectrum of heavy quarkonia}

For the mass spectrum of heavy quarkonia $\left(Q_{1} \bar{Q}_{2}\right)$ we present our results in Tables III. Again it is interesting to consider the limit of heavy quark masses. Here we follow the idea suggested in [5] , and express the longitudinal momentum fractions through the $z$-component of the internal momentum $\mathbf{k}=\left(\mathbf{k}_{\perp}, k_{z}\right)$ as (see also [45]):

$$
x=\frac{e_{1}+k_{z}}{e_{1}+e_{2}}, \quad 1-x=\frac{e_{2}-k_{z}}{e_{1}+e_{2}},
$$

where $e_{i}=\sqrt{m_{Q_{i}}^{2}+\mathbf{k}^{2}}$ and $\mathbf{k}^{2}=\mathbf{k}_{\perp}^{2}+k_{z}^{2}$. When considering the heavy quark limit $m_{Q_{1}}, m_{Q_{2}} \gg \mathbf{k}_{\perp}, k_{z}$ we get

$$
x=\frac{m_{Q_{1}}+k_{z}}{m_{Q_{1}}+m_{Q_{2}}}+\mathcal{O}\left(1 / m_{Q}^{2}\right), \quad 1-x=\frac{m_{Q_{2}}-k_{z}}{m_{Q_{1}}+m_{Q_{2}}}+\mathcal{O}\left(1 / m_{Q}^{2}\right) .
$$

Hence, we have

$$
\frac{m_{Q_{1}}^{2}}{x}+\frac{m_{Q_{2}}^{2}}{1-x}=\left(m_{Q_{1}}+m_{Q_{2}}\right)^{2}+\mathcal{O}(1) .
$$

Therefore, the leading term of the integral containing the longitudinal mode is simply given by $\left(m_{Q_{1}}+m_{Q_{2}}\right)^{2}$ which is the leading contribution to the mass squared of the heavy quarkonia. This means that we correctly reproduce an expansion of the heavy quarkonia mass in the heavy quark limit:

$$
M_{Q_{1} \bar{Q}_{2}}=m_{Q_{1}}+m_{Q_{2}}+E+\mathcal{O}\left(1 / m_{Q_{1,2}}\right),
$$

where $E$ is the binding energy. Numerical values for the quarkonia masses are shown in Table III, according to the $n^{2 S+1} L_{J}$ classification.

A graphical summary of our results is given in Figs.1-6 where we display the calculated meson mass spectra for light (Figs.1 and 2), heavy-light (Figs.3 and 4) and heavy quarkonia (Figs.5 and 6). Because of the inclusion of the color Coulomb potential the lines are bent down for low values of $L$ or $n$ and therefore deviate from the linear behavior of Regge-trajectories. We indicate the available data (central values by black circles and sizable error bars by vertical lines). Here we find that the calculated mass spectra are in agreement with predictions of other holographic models (see e.g. Refs. [14, 15, 20]). The improvement of the present approach is that a consistent description of light, heavylight and double-heavy mesons is achieved within the same holographic model. For comparison, in the literature so far different types of mesons have been considered separately. As already mentioned, we use a universal value for the dilaton parameter $\kappa$. However, adapted values of $\kappa$ for different types of mesons leads of course to a better fit to the data.

\section{Leptonic and radiative meson decay constants}

In the following we define further fundamental quantities of mesons, which are calculated in the present paper decay constants of pseudoscalar $\left(f_{P}\right)$

$$
\left\langle 0\left|\bar{q}_{2}(0) \gamma^{\mu} \gamma^{5} q_{1}(0)\right| M_{P}(P)\right\rangle=i P^{\mu} f_{P},
$$


and vector $\left(f_{V}\right)$ mesons

$$
\left\langle 0\left|\bar{q}_{2}(0) \gamma^{\mu} q_{1}(0)\right| M_{V}(P, \lambda)\right\rangle=\epsilon^{\mu}(P, \lambda) M_{V} f_{V} .
$$

The definitions of the meson Fock states are given in Appendix $\mathrm{A}$

For convenience we determine the decay constants restricting to $\mu=+$ and spin projection $\lambda=0$ for the vector mesons. In this case Eqs. (45) and (46) are simply reduced to

$$
\begin{aligned}
& \left\langle 0\left|\bar{q}_{2}(0) \gamma^{+} \gamma^{5} q_{1}(0)\right| P\right\rangle=P^{+} f_{P}, \\
& \left\langle 0\left|\bar{q}_{2}(0) \gamma^{+} q_{1}(0)\right| V(P, \lambda=0)\right\rangle=P^{+} f_{V} .
\end{aligned}
$$

Finally, we get for the couplings of the ground state mesons:

$$
\begin{aligned}
f_{P} & =f_{V}=2 \sqrt{6} \int_{0}^{1} d x \int \frac{d^{2} \mathbf{k}_{\perp}}{16 \pi^{3}} \psi_{q_{1} \bar{q}_{2}}\left(x, \mathbf{k}_{\perp}\right) f\left(x, m_{1}, m_{2}\right)=\sqrt{\frac{6}{\pi}} \int_{0}^{1} d x \tilde{\psi}_{q_{1} \bar{q}_{2}}\left(x, \mathbf{b}_{\perp}=0\right) f\left(x, m_{1}, m_{2}\right) \\
& =\kappa \frac{\sqrt{6}}{\pi} \int_{0}^{1} d x \sqrt{x(1-x)} f\left(x, m_{1}, m_{2}\right)
\end{aligned}
$$

In the case of massless quarks $f_{P}$ and $f_{V}$ are proportional to the dilaton scale parameter $\kappa$ :

$$
f_{P}=f_{V}=\frac{\kappa \sqrt{6}}{8}
$$

In the heavy quark limit $\left(m_{Q} \rightarrow \infty\right)$ the scaling of the leptonic decay constants of heavy-light mesons is in agreement with HQET:

$$
f_{P}^{\mathrm{HQL}}=f_{V}^{\mathrm{HQL}}=\kappa \frac{\sqrt{6}}{\pi} \sqrt{\frac{m_{q}}{m_{Q}}} \frac{\int_{0}^{\infty} d z e^{-\frac{r}{2}\left(z+\frac{1}{z}\right)}}{\left[\int_{0}^{\infty} d z e^{-r\left(z+\frac{1}{z}\right)}\right]^{1 / 2}} \sim \frac{1}{\sqrt{m_{Q}}} .
$$

Again, as in the case of the mass spectrum of heavy-light mesons, it is sufficient to propose the following scaling of dimensional parameters in our holographic approach: $\kappa \sim \mathcal{O}(1)$ and $\lambda_{q Q} \sim \mathcal{O}\left(\sqrt{m_{Q}}\right)$.

When dealing with vector mesons with hidden flavor one should also include the flavor factor $c_{V}$

$$
c_{V}= \begin{cases}1 / \sqrt{2}, & V=\rho^{0} \\ 2 / 3, & V=J / \psi \\ 1 / 3, & V=\phi, \Upsilon \\ 1 /(3 \sqrt{2}), & V=\omega\end{cases}
$$

which arise from the flavor structure of the vector mesons

$$
\rho^{0}=\frac{1}{\sqrt{2}}(\bar{u} u-\bar{d} d), \quad \omega=\frac{1}{\sqrt{2}}(\bar{u} u+\bar{d} d), \quad \phi=-\bar{s} s, \quad J / \psi=\bar{c} c, \quad \Upsilon=-\bar{b} b
$$

and the structure of the corresponding electromagnetic quark currents

$$
\begin{aligned}
V_{\rho, \omega}^{\mu} & =e_{u} \bar{u} \gamma^{\mu} u+e_{d} \bar{d} \gamma^{\mu} d \\
V_{\phi, J / \psi, \Upsilon}^{\mu} & =e_{q} \bar{q} \gamma^{\mu} q, \text { with } q=s, c, b .
\end{aligned}
$$

Our results for $f_{P}$ and $f_{V}$ are presented in Tables IV-VI. Note that with the universal value of the dilaton scale parameter $\kappa=550 \mathrm{MeV}$ the data for the coupling constants of the light mesons can be well reproduced. For heavylight mesons we need a bit larger value for the parameter $\kappa$, because the leptonic decay constants are proportional to $\kappa$. For the description of the leptonic decay constants of heavy quarkonia states we need an even larger value for $\kappa$. In particular, it should be roughly 2,3 and 4 times larger for $c \bar{c}, c \bar{b}$ and $b \bar{b}$ states, respectively, than the unified value of $550 \mathrm{MeV}$. 


\section{CONCLUSIONS}

In conclusion, we present an analysis of the mass spectrum and decay properties of light, heavy-light mesons and heavy quarkonia in an holographic soft-wall model using conventional sign of the dilaton profile with $\phi(z)=\kappa^{2} z^{2}$. In our calculations we consider in addition one-gluon exchange and hyperfine-splitting corrections phenomenologically by modifying the potential. We show that the obtained results for heavy-light mesons are consistent with constraints imposed by HQET. In future work we plan to improve the description of meson data and also to extend our formalism to baryons.

\section{Acknowledgments}

The authors thank Stan Brodsky, Guy de Téramond, Herry Kwee and Oleg Andreev for useful discussions. This work was supported by the DFG under Contract No. FA67/31-2 and No. GRK683, by FONDECYT (Chile) under Grant No. 1100287. This research is also part of the European Community-Research Infrastructure Integrating Activity "Study of Strongly Interacting Matter" (HadronPhysics2, Grant Agreement No. 227431), Russian President grant "Scientific Schools" No. 3400.2010.2, Federal Targeted Program "Scientific and scientific-pedagogical personnel of innovative Russia" Contract No. 02.740.11.0238. A. V. acknowledges the financial support from FONDECYT (Chile) Grant No. 3100028.

\section{Appendix A: Light-front meson Fock states}

Here we define the Fock states of pseudoscalar $(P)$, scalar $(S)$, vector $(V)$ and axial $(A)$ mesons, restricting to the valence quark-antiquark contribution only. The corresponding mesonic eigenstates with momentum $P=$ $\left(P^{+}, P^{-}, \mathbf{P}_{\perp}\right)$ are given by

$$
\left|M_{P, S}(P)\right\rangle=\frac{2 P^{+}}{\sqrt{2 N_{c}}} \int_{0}^{1} d x \int \frac{d^{2} \mathbf{k}_{\perp}}{16 \pi^{3}} \psi_{q_{1} \bar{q}_{2}}\left(x, \mathbf{k}_{\perp}\right)\left[d_{q_{1} \uparrow}^{\dagger a}\left(p_{1}\right) b_{q_{2} \downarrow}^{\dagger a}\left(p_{2}\right)-d_{q_{1} \downarrow}^{\dagger a}\left(p_{1}\right) b_{q_{2} \uparrow}^{\dagger a}\left(p_{2}\right)\right]|0\rangle
$$

and

$$
\left|M_{V, A}(P, \lambda)\right\rangle=\frac{2 P^{+}}{\sqrt{2 N_{c}}} \int_{0}^{1} d x \int \frac{d^{2} \mathbf{k}_{\perp}}{16 \pi^{3}} \psi_{q_{1} \bar{q}_{2}}\left(x, \mathbf{k}_{\perp}\right) \begin{cases}d_{q \uparrow}^{\dagger a}(x P) b_{q \uparrow}^{\dagger a}((1-x) P)|0\rangle \sqrt{2}, & \lambda=+1 \\ d_{q \uparrow}^{\dagger a}(x P) b_{q \downarrow}^{\dagger a}((1-x) P)+d_{q \downarrow}^{\dagger a}(x P) b_{q \uparrow}^{\dagger a}((1-x) P)|0\rangle, & \lambda=0 \\ d_{q \downarrow}^{\dagger a}(x P) b_{q \downarrow}^{\dagger a}((1-x) P)|0\rangle \sqrt{2}, & \lambda=-1\end{cases}
$$

with

$$
\left(p_{1}\right)=\left(x P^{+}, x \mathbf{P}_{\perp}+\mathbf{k}_{\perp}\right), \quad\left(p_{2}\right)=\left((1-x) P^{+},(1-x) \mathbf{P}_{\perp}-\mathbf{k}_{\perp}\right) .
$$

Here $a$ is the color index and $N_{c}=3$ is the number of colors. In the expansion (A1) of the meson state over the basis of noninteracting n-particles states we restrict to the two-quark valence state. Notice that above formulas can be extended easily to the case of mesons with more nontrivial flavor structure, e.g. including mixing of nonstrange and strange quark components. Also one can consider pure glueball states and their mixing with quarkonia.

The operators $b(d)$ obey the anticommutation relations:

$$
\left\{b^{a}(p), b^{\dagger a^{\prime}}\left(p^{\prime}\right\}=\left\{d^{a}(p), d^{\dagger a^{\prime}}\left(p^{\prime}\right\}=(2 \pi)^{3} \delta^{a a^{\prime}} \delta\left(p^{+}-p^{\prime+}\right) \delta^{(2)}\left(\mathbf{p}_{\perp}-\mathbf{p}_{\perp}^{\prime}\right) .\right.\right.
$$

The states $\left|M_{H}(P)\right\rangle$ and momentum LFWF are normalized according to

$$
\begin{gathered}
\left\langle M_{P}\left(P^{\prime}\right) \mid M_{P}(P)\right\rangle=2 P^{+}(2 \pi)^{3} \delta\left(P^{+}-P^{\prime+}\right) \delta^{(2)}\left(\mathbf{P}_{\perp}-\mathbf{P}_{\perp}^{\prime}\right), \\
\left\langle M_{V}\left(P^{\prime}, \lambda^{\prime}\right) \mid M_{V}(P, \lambda)\right\rangle=2 P^{+}(2 \pi)^{3} \delta\left(P^{+}-P^{\prime+}\right) \delta^{(2)}\left(\mathbf{P}_{\perp}-\mathbf{P}_{\perp}^{\prime}\right) \delta_{\lambda \lambda^{\prime}},
\end{gathered}
$$

and

$$
\int_{0}^{1} d x \int \frac{d^{2} \mathbf{k}_{\perp}}{16 \pi^{3}}\left|\psi_{\bar{q}_{2} q_{1}}\left(x, \mathbf{k}_{\perp}\right)\right|^{2}=1
$$


The polarization vectors $\epsilon^{\mu}(P, \lambda)$ in the light-cone representation read as

$$
\epsilon^{\mu}(P, \lambda)= \begin{cases}\left(\frac{P^{+}}{M_{V}}, \frac{\mathbf{P}_{\perp}^{2}-M_{V}^{2}}{M_{V} P^{+}}, \frac{\mathbf{P}_{\perp}}{M_{V}}\right), & \lambda=0 \\ \left(0, \frac{2 \mathbf{e}_{\perp}( \pm 1) \mathbf{P}_{\perp}}{P^{+}}, \mathbf{e}_{\perp}( \pm 1)\right), & \lambda= \pm 1\end{cases}
$$

with $\mathbf{e}_{\perp}( \pm 1)=\mp \frac{(1, \pm i)}{\sqrt{2}}$

Note that the normalization condition (A6) is approximately valid only for mesons, where the two-parton quarkantiquark component is dominant. As we stressed before (also see discussion in Ref. [31]), this is not the case for the pion, where the probability of the valence quark-antiquark component is less than 1 [see also Eq. (15)].

\section{Appendix B: Evaluation of integrals in the heavy quark limit}

We evaluate the integral in the r.h.s. of Eq. (37) with

$$
J=\int_{0}^{1} d x\left(\frac{m_{q}^{2}}{x}+\frac{m_{Q}^{2}}{1-x}\right) f^{2}\left(x, m_{q}, m_{Q}\right)=\frac{\int_{0}^{1} d x\left(\frac{m_{q}^{2}}{x}+\frac{m_{Q}^{2}}{1-x}\right) \exp \left(-\frac{m_{q}^{2}}{\lambda_{q Q}^{2} x}+\frac{m_{Q}^{2}}{\lambda_{q Q}^{2}(1-x)}\right)}{\int_{0}^{1} d x \exp \left(-\frac{m_{q}^{2}}{\lambda_{q Q}^{2} x}+\frac{m_{Q}^{2}}{\lambda_{q Q}^{2}(1-x)}\right)} .
$$

Scaling the variable $x=z m_{q} / m_{Q}$ and then performing an expansion in powers of $1 / m_{Q}$ :

$$
J=m_{Q}^{2}+m_{Q} m_{q} \frac{\int_{0}^{\infty} d z\left(z+\frac{1}{z}\right) \exp \left(-\frac{m_{q} m_{Q}}{\lambda_{q Q}^{2}}\left(z+\frac{1}{z}\right)\right)}{\int_{0}^{\infty} d z \exp \left(-\frac{m_{q} m_{Q}}{\lambda_{q Q}^{2}}\left(z+\frac{1}{z}\right)\right)}+\mathcal{O}(1)
$$

where the parameter $\lambda_{q Q}$ scales as $\sqrt{m_{Q}}$. Such a scaling of $\kappa$ is consistent with the scaling of the leptonic coupling constants of heavy-light mesons (see Sec. IIID). For convenience, we introduce the parameter $r=m_{q} m_{Q} / \lambda_{q Q}^{2}$. Finally, the expansion of the heavy-light meson mass reads

$$
M_{q Q}=m_{Q}+\bar{\Lambda}+\mathcal{O}\left(1 / m_{Q}\right)
$$

where

$$
\bar{\Lambda}=m_{q} I
$$

and

$$
I=\frac{1}{2} \frac{\int_{0}^{\infty} d z\left(z+\frac{1}{z}\right) e^{-r\left(z+\frac{1}{z}\right)}}{\int_{0}^{\infty} d z e^{-r\left(z+\frac{1}{z}\right)}}
$$

[1] S. J. Brodsky and G. F. de Teramond, Phys. Lett. B 582, 211 (2004); arXiv:hep-th/0310227. S. J. Brodsky and G. F. de Teramond, Phys. Rev. Lett. 96, 201601 (2006) arXiv:hep-ph/0602252.

[2] S. J. Brodsky and G. F. de Teramond, Phys. Rev. D 77, 056007 (2008) arXiv:0707.3859 [hep-ph]].

[3] S. J. Brodsky and G. F. de Teramond, arXiv:0802.0514 [hep-ph].

[4] S. J. Brodsky and G. F. de Teramond, Phys. Rev. D 78, 025032 (2008) arXiv:0804.0452 [hep-ph]].

[5] G. F. de Teramond, Invited Talk at the Third Workshop of the APS Topical Group in Hadron Physics "GHP2009", http://www.fz-juelich.de/ikp/ghp2009/Program.shtml

[6] S. Brodsky, Invited Talk "Light-Front Holography and Novel QCD Phenomena" at the PKU-RBRC Workshop on Transverse Spin Physics. CHEP, Peking University, July 3, 2008.

[7] S. J. Brodsky and R. Shrock, arXiv:0905.1151 [hep-th]. 
[8] S. J. Brodsky, Invited Talk at the JTI Workshop on Dynamics of Symmetry Breaking, ANL, IL, http://www.slac.stanford.edu/grp/th/recentlectures.html

[9] J. M. Maldacena, Adv. Theor. Math. Phys. 2, 231 (1998) [Int. J. Theor. Phys. 38, 1113 (1999)] arXiv:hep-th/9711200]; S. S. Gubser, I. R. Klebanov and A. M. Polyakov, Phys. Lett. B 428, 105 (1998) arXiv:hep-th/9802109; E. Witten, Adv. Theor. Math. Phys. 2, 253 (1998) arXiv:hep-th/9802150.

[10] I. R. Klebanov and J. M. Maldacena, Phys. Today 62, 28 (2009).

[11] J. Polchinski and M. J. Strassler, Phys. Rev. Lett. 88, 031601 (2002) arXiv:hep-th/0109174.

[12] H. Boschi-Filho and N. R. F. Braga, JHEP 0305, 009 (2003) arXiv:hep-th/0212207.

[13] G. F. de Teramond and S. J. Brodsky, Phys. Rev. Lett. 94, 201601 (2005) arXiv:hep-th/0501022;

[14] J. Erlich, E. Katz, D. T. Son and M. A. Stephanov, Phys. Rev. Lett. 95, 261602 (2005) arXiv:hep-ph/0501128].

[15] L. Da Rold and A. Pomarol, Nucl. Phys. B 721, 79 (2005) arXiv:hep-ph/0501218.

[16] K. Ghoroku, N. Maru, M. Tachibana and M. Yahiro, Phys. Lett. B 633, 602 (2006) arXiv:hep-ph/0510334.

[17] E. Katz, A. Lewandowski and M. D. Schwartz, Phys. Rev. D 74, 086004 (2006) arXiv:hep-ph/0510388.

[18] G. F. de Teramond and S. J. Brodsky, Phys. Rev. Lett. 102, 081601 (2009) arXiv:0809.4899 [hep-ph]].

[19] H. R. Grigoryan and A. V. Radyushkin, Phys. Lett. B 650, 421 (2007) arXiv:hep-ph/0703069; H. R. Grigoryan and A. V. Radyushkin, Phys. Rev. D 76, 115007 (2007) arXiv:0709.0500 [hep-ph]].

[20] A. Karch, E. Katz, D. T. Son and M. A. Stephanov, Phys. Rev. D 74, 015005 (2006) arXiv:hep-ph/0602229].

[21] O. Andreev, Phys. Rev. D 73, 107901 (2006) arXiv:hep-th/0603170; O. Andreev and V. I. Zakharov, Phys. Rev. D 74, 025023 (2006) arXiv:hep-ph/0604204.

[22] H. Forkel, M. Beyer and T. Frederico, JHEP 0707, 077 (2007) arXiv:0705.1857 [hep-ph]]; H. Forkel, M. Beyer and T. Frederico, Int. J. Mod. Phys. E 16, 2794 (2007) arXiv:0705.4115 [hep-ph]]; H. Forkel, Phys. Rev. D 78, 025001 (2008) arXiv:0711.1179 [hep-ph]].

[23] H. R. Grigoryan and A. V. Radyushkin, Phys. Rev. D 76, 095007 (2007) arXiv:0706.1543 [hep-ph]].

[24] U. Gursoy and E. Kiritsis, JHEP 0802, 032 (2008) arXiv:0707.1324 [hep-th]]; U. Gursoy, E. Kiritsis and F. Nitti, JHEP 0802, 019 (2008) arXiv:0707.1349 [hep-th]]; W. de Paula, T. Frederico, H. Forkel and M. Beyer, Phys. Rev. D 79, 075019 (2009) arXiv:0806.3830 [hep-ph]].

[25] R. Casero, E. Kiritsis and A. Paredes, Nucl. Phys. B 787, 98 (2007) arXiv:hep-th/0702155; O. Bergman, S. Seki and J. Sonnenschein, JHEP 0712, 037 (2007) arXiv:0708.2839 [hep-th]].

[26] H. J. Kwee and R. F. Lebed, Phys. Rev. D 77, 115007 (2008) arXiv:0712.1811 [hep-ph]].

[27] P. Colangelo, F. De Fazio, F. Jugeau and S. Nicotri, Phys. Lett. B 652, 73 (2007) arXiv:hep-ph/0703316]; P. Colangelo, F. De Fazio, F. Giannuzzi, F. Jugeau and S. Nicotri, Phys. Rev. D 78, 055009 (2008) [arXiv:0807.1054 [hep-ph]].

[28] A. Vega and I. Schmidt, Phys. Rev. D 78, 017703 (2008) arXiv:0806.2267 [hep-ph]].

[29] A. Vega and I. Schmidt, Phys. Rev. D 79, 055003 (2009) arXiv:0811.4638 [hep-ph]].

[30] T. Gherghetta, J. I. Kapusta and T. M. Kelley, Phys. Rev. D 79, 076003 (2009) arXiv:0902.1998 [hep-ph]].

[31] A. Vega, I. Schmidt, T. Branz, T. Gutsche and V. E. Lyubovitskij, Phys. Rev. D 80, 055014 (2009) arXiv:0906.1220 [hep-ph]].

[32] B. Galow, E. Megias, J. Nian and H. J. Pirner, Nucl. Phys. B 834, 330 (2010) arXiv:0911.0627 [hep-ph]]; J. Nian and H. J. Pirner, Nucl. Phys. A 833, 119 (2010) arXiv:0908.1330 [hep-ph]].

[33] Y. Q. Sui, Y. L. Wu, Z. F. Xie and Y. B. Yang, Phys. Rev. D 81, 014024 (2010) arXiv:0909.3887 [hep-ph]].

[34] Z. Abidin and C. E. Carlson, Phys. Rev. D 79, 115003 (2009) arXiv:0903.4818 [hep-ph]].

[35] M. Fujita, K. Fukushima, T. Misumi and M. Murata, Phys. Rev. D 80, 035001 (2009) arXiv:0903.2316 [hep-ph]]; M. Fujita, T. Kikuchi, K. Fukushima, T. Misumi and M. Murata, Phys. Rev. D 81, 065024 (2010) arXiv:0911.2298 [hep-ph]].

[36] H. R. Grigoryan, P. M. Hohler and M. A. Stephanov, Phys. Rev. D 82, 026005 (2010) arXiv:1003.1138 [hep-ph].

[37] S. S. Afonin, arXiv:1001.3105 [hep-ph].

[38] J. Babington, J. Erdmenger, N. J. Evans, Z. Guralnik and I. Kirsch, Phys. Rev. D 69, 066007 (2004) arXiv:hep-th/0306018.

[39] M. N. Sergeenko, Phys. Atom. Nucl. 56, 365 (1993) [Yad. Fiz. 56, 140 (1993)]; M. N. Sergeenko, Z. Phys. C 64 (1994) 315.

[40] S. S. Gershtein, A. K. Likhoded and A. V. Luchinsky, Phys. Rev. D 74, 016002 (2006) arXiv:hep-ph/0602048.

[41] S. G. Zhou and H. C. Pauli, J. Phys. G 30, 983 (2004) arXiv:hep-ph/0311305.

[42] M. Karliner and H. J. Lipkin, Phys. Lett. B 650, 185 (2007) arXiv:hep-ph/0608004. M. Karliner, B. Keren-Zur, H. J. Lipkin and J. L. Rosner, Annals Phys. 324, 2 (2009) arXiv:0804.1575 [hep-ph]].

[43] A. M. Badalian, A. I. Veselov and B. L. G. Bakker, Phys. Rev. D 70, 016007 (2004); D. Ebert, R. N. Faustov and V. O. Galkin, Phys. Rev. D 79, 114029 (2009) arXiv:0903.5183 [hep-ph]].

[44] M. Neubert, Phys. Rept. 245, 259 (1994) arXiv:hep-ph/9306320.

[45] W. Jaus, Phys. Rev. D 41, 3394 (1990).

[46] K. Nakamura et al. (Particle Data Group), J. Phys. G 37, 075021 (2010).

[47] T. W. Chiu and T. H. Hsieh (TWQCD Collaboration), PoS LAT2006, 180 (2007) arXiv:0704.3495 [hep-lat]].

[48] D. Becirevic, P. Boucaud, J. P. Leroy, V. Lubicz, G. Martinelli, F. Mescia and F. Rapuano, Phys. Rev. D 60, 074501 (1999) arXiv:hep-lat/9811003.

[49] C. Aubin et al., Phys. Rev. Lett. 95, 122002 (2005) arXiv:hep-lat/0506030. 
TABLE I: Masses of light mesons

\begin{tabular}{|l|c|c|c|l|l|l|l|}
\hline Meson & $n$ & $L$ & $S$ & \multicolumn{5}{|c|}{ Mass $[\mathrm{MeV}]$} \\
\hline$\pi$ & 0 & $0,1,2,3$ & 0 & $M_{\pi(140)}=140$ & $M_{b_{1}(1235)}=1355$ & $M_{\pi_{2}(1670)}=1777$ & $M_{\pi_{4}}=2099$ \\
\hline$\pi$ & $0,1,2,3$ & 0 & 0 & $M_{\pi(140)}=140$ & $M_{\pi(1300)}=1355$ & $M_{\pi(1800)}=1777$ & $M_{\pi(4 s)}=2099$ \\
\hline$K$ & 0 & $0,1,2,3$ & 0 & $M_{K}=495$ & $M_{K_{1}(1270)}=1505$ & $M_{K_{2}(1770)}=1901$ & $M_{K_{3}}=2207$ \\
\hline$\eta$ & $0,1,2,3$ & 0 & 0 & $M_{\eta(1 s)}=544$ & $M_{\eta(2 s)}=1552$ & $M_{\eta(3 s)}=1946$ & $M_{\eta(4 s)}=2248$ \\
\hline$f_{0}[\bar{n} n]$ & $0,1,2,3$ & 1 & 1 & $M_{f_{0}(1 p)}=1114$ & $M_{f_{0}(2 p)}=1600$ & $M_{f_{0}(3 p)}=1952$ & $M_{f_{0}(4 p)}=2244$ \\
\hline$f_{0}[\bar{s} s]$ & $0,1,2,3$ & 1 & 1 & $M_{f_{0}(1 p)}=1304$ & $M_{f_{0}(2 p)}=1762$ & $M_{f_{0}(3 p)}=2093$ & $M_{f_{0}(4 p)}=2372$ \\
\hline$a_{0}(980)$ & $0,1,2,3$ & 1 & 1 & $M_{a_{0}(1 p)}=1114$ & $M_{a_{0}(2 p)}=1600$ & $M_{a_{0}(3 p)}=1952$ & $M_{a_{0}(4 p)}=2372$ \\
\hline$\rho(770)$ & $0,1,2,3$ & 0 & 1 & $M_{\rho(770)}=804$ & $M_{\rho(1450)}=1565$ & $M_{\rho_{(1700)}}=1942$ & $M_{\rho(4 s)}=2240$ \\
\hline$\rho(770)$ & 0 & $0,1,2,3$ & 1 & $M_{\rho(770)}=804$ & $M_{a_{2}(1320)}=1565$ & $M_{\rho_{3}(1690)}=1942$ & $M_{a_{4}(2040)}=2240$ \\
\hline$\omega(782)$ & $0,1,2,3$ & 0 & 1 & $M_{\omega(782)}=804$ & $M_{\omega(1420)}=1565$ & $M_{\omega(1650)}=1942$ & $M_{\omega(4 s)}=2240$ \\
\hline$\omega(782)$ & 0 & $0,1,2,3$ & 1 & $M_{\omega(782)}=804$ & $M_{f_{2}(1270)}=1565$ & $M_{\omega_{3}(1670)}=1942$ & $M_{f_{4}(2050)}=2240$ \\
\hline$\phi(1020)$ & $0,1,2,3$ & 0 & 1 & $M_{\phi(1 s)}=1019$ & $M_{\phi(2 s)}=1818$ & $M_{\phi(3 s)}=2170$ & $M_{\phi(4 s)}=2447$ \\
\hline$a_{1}(1260)$ & $0,1,2,3$ & 1 & 1 & $M_{a_{1}(1 p)}=1358$ & $M_{a_{1}(2 p)}=1779$ & $M_{a_{1}(3 p)}=2101$ & $M_{a_{1}(4 p)}=2375$ \\
\hline
\end{tabular}

TABLE II: Masses of heavy-light mesons

\begin{tabular}{|l|l|l|c|c|c|c|c|c|}
\hline Meson & $J^{\mathrm{P}}$ & $n$ & $L$ & $S$ & \multicolumn{4}{|c|}{ Mass $[\mathrm{MeV}]$} \\
\hline$D(1870)$ & $0^{-}$ & 0 & $0,1,2,3$ & 0 & 1857 & 2435 & 2696 & 2905 \\
\hline$D^{*}(2010)$ & $1^{-}$ & 0 & $0,1,2,3$ & 1 & 2015 & 2547 & 2797 & 3000 \\
\hline$D_{s}(1969)$ & $0^{-}$ & 0 & $0,1,2,3$ & 0 & 1963 & 2621 & 2883 & 3085 \\
\hline$D_{s}^{*}(2107)$ & $1^{-}$ & 0 & $0,1,2,3$ & 1 & 2113 & 2725 & 2977 & 3173 \\
\hline$B(5279)$ & $0^{-}$ & 0 & $0,1,2,3$ & 0 & 5279 & 5791 & 5964 & 6089 \\
\hline$B^{*}(5325)$ & $1^{-}$ & 0 & $0,1,2,3$ & 1 & 5336 & 5843 & 6015 & 6139 \\
\hline$B_{s}(5366)$ & $0^{-}$ & 0 & $0,1,2,3$ & 0 & 5360 & 5941 & 6124 & 6250 \\
\hline$B_{s}^{*}(5413)$ & $1^{-}$ & 0 & $0,1,2,3$ & 1 & 5416 & 5992 & 6173 & 6298 \\
\hline
\end{tabular}

TABLE III: Masses of heavy quarkonia $c \bar{c}, b \bar{b}$ and $c \bar{b}$

\begin{tabular}{|l|l|c|c|c|c|c|c|c|}
\hline Meson & $J^{\mathrm{P}}$ & $n$ & $L$ & $S$ & \multicolumn{5}{|c|}{ Mass $[\mathrm{MeV}]$} \\
\hline$\eta_{c}(2980)$ & $0^{-}$ & $0,1,2,3$ & 0 & 0 & 2997 & 3717 & 3962 & 4141 \\
\hline$\psi(3097))$ & $1^{-}$ & $0,1,2,3$ & 0 & 1 & 3097 & 3798 & 4038 & 4213 \\
\hline$\chi_{c 0}(3415)$ & $0^{+}$ & $0,1,2,3$ & 1 & 1 & 3635 & 3885 & 4067 & 4226 \\
\hline$\chi_{c 1}(3510)$ & $1^{+}$ & $0,1,2,3$ & 1 & 1 & 3718 & 3963 & 4141 & 4297 \\
\hline$\chi_{c 2}(3555)$ & $2^{+}$ & $0,1,2,3$ & 1 & 1 & 3798 & 4038 & 4213 & 4367 \\
\hline$\eta_{b}(9390)$ & $0^{-}$ & $0,1,2,3$ & 0 & 0 & 9428 & 10190 & 10372 & 10473 \\
\hline$\Upsilon(9460)$ & $1^{-}$ & $0,1,2,3$ & 0 & 1 & 9460 & 10219 & 10401 & 10502 \\
\hline$\chi_{b 0}(9860)$ & $0^{+}$ & $0,1,2,3$ & 1 & 1 & 10160 & 10343 & 10444 & 10521 \\
\hline$\chi_{b 1}(9893)$ & $1^{+}$ & $0,1,2,3$ & 1 & 1 & 10190 & 10372 & 10473 & 10550 \\
\hline$\chi_{b 2}(9912)$ & $2^{+}$ & $0,1,2,3$ & 1 & 1 & 10219 & 10401 & 10502 & 10579 \\
\hline$B_{c}(6276)$ & $0^{-}$ & $0,1,2,3$ & 0 & 0 & 6276 & 6911 & 7092 & 7209 \\
\hline
\end{tabular}


TABLE IV: Decay constants $f_{P}$ of pseudoscalar mesons in $\mathrm{MeV}$

\begin{tabular}{|l|c|c|}
\hline Meson & Data [46] & Our \\
\hline$\pi^{-}$ & $130.4 \pm 0.03 \pm 0.2$ & 131 \\
\hline$K^{-}$ & $156.1 \pm 0.2 \pm 0.8$ & 155 \\
\hline$D^{+}$ & $206.7 \pm 8.9$ & 167 \\
\hline$D_{s}^{+}$ & $257.5 \pm 6.1$ & 170 \\
\hline$B^{-}$ & $193 \pm 11$ & 139 \\
\hline$B_{s}^{0}$ & $253 \pm 8 \pm 7$ & 144 \\
\hline$B_{c}$ & $489 \pm 5 \pm 3[47]$ & 159 \\
\hline
\end{tabular}

TABLE V: Decay constants $f_{V}$ of vector mesons with open flavor in $\mathrm{MeV}$

\begin{tabular}{|l|c|c|}
\hline Meson & Data & Our \\
\hline$\rho^{+}$ & $210.5 \pm 0.6[46]$ & 170 \\
\hline$D^{*}$ & $245 \pm 20_{-2}^{+3}[48]$ & 167 \\
\hline$D_{s}^{*}$ & $272 \pm 16_{-20}^{+3}[49]$ & 170 \\
\hline$B^{*}$ & $196 \pm 24_{-2}^{+39}[48]$ & 139 \\
\hline$B_{s}^{*}$ & $229 \pm 20_{-16}^{+41}[48]$ & 144 \\
\hline
\end{tabular}

TABLE VI: Decay constants $f_{V}$ of vector mesons with hidden flavor in $\mathrm{MeV}$

\begin{tabular}{|l|c|c|}
\hline Meson & Data [46] & Our \\
\hline$\rho^{0}$ & $154.7 \pm 0.7$ & 120 \\
\hline$\omega$ & $45.8 \pm 0.8$ & 40 \\
\hline$\phi$ & $76 \pm 1.2$ & 58 \\
\hline$J / \psi$ & $277.6 \pm 4$ & 116 \\
\hline$\Upsilon(1 s)$ & $238.5 \pm 5.5$ & 56 \\
\hline
\end{tabular}




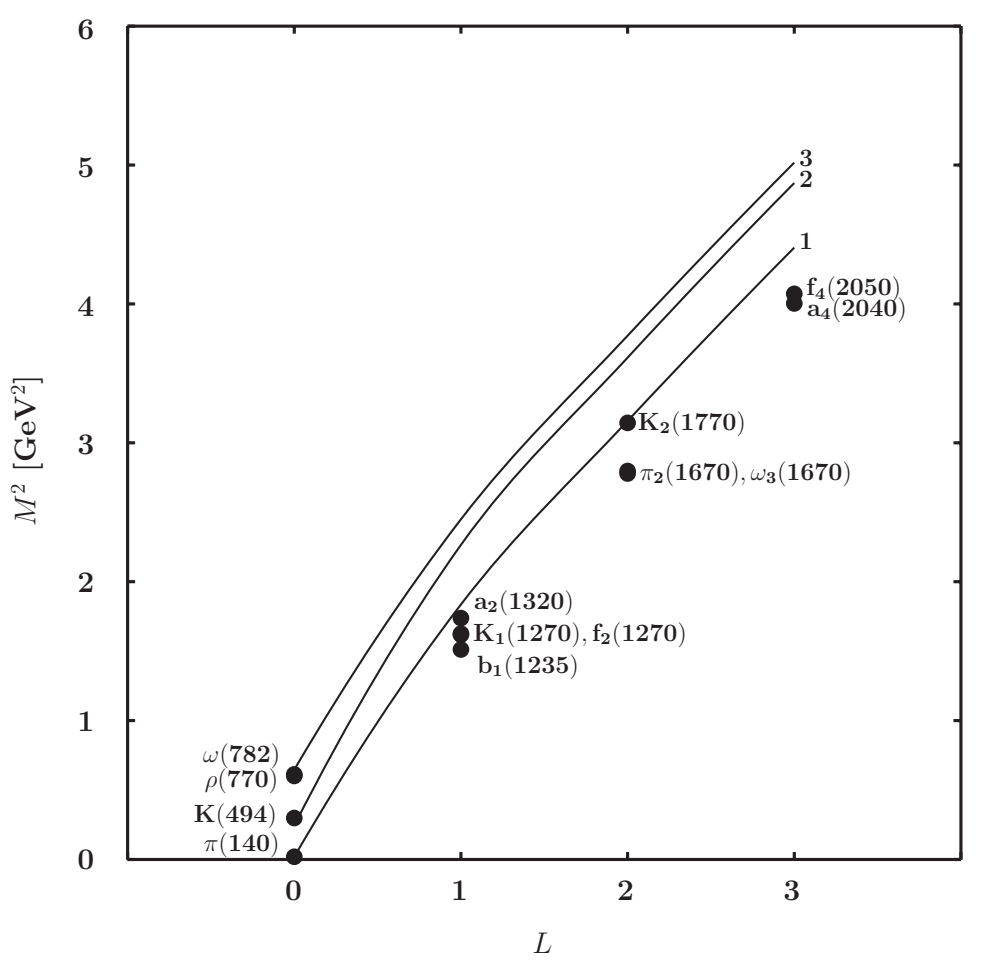

FIG. 1: Mass spectrum of $\pi, K, \rho$ and $\omega$ mesons in dependence on $L$. Central values of data are indicated by black circles. The numbers mark the corresponding family trajectories: $1-\pi$ mesons; $2-K$ mesons; $3-\rho, \omega$ mesons.

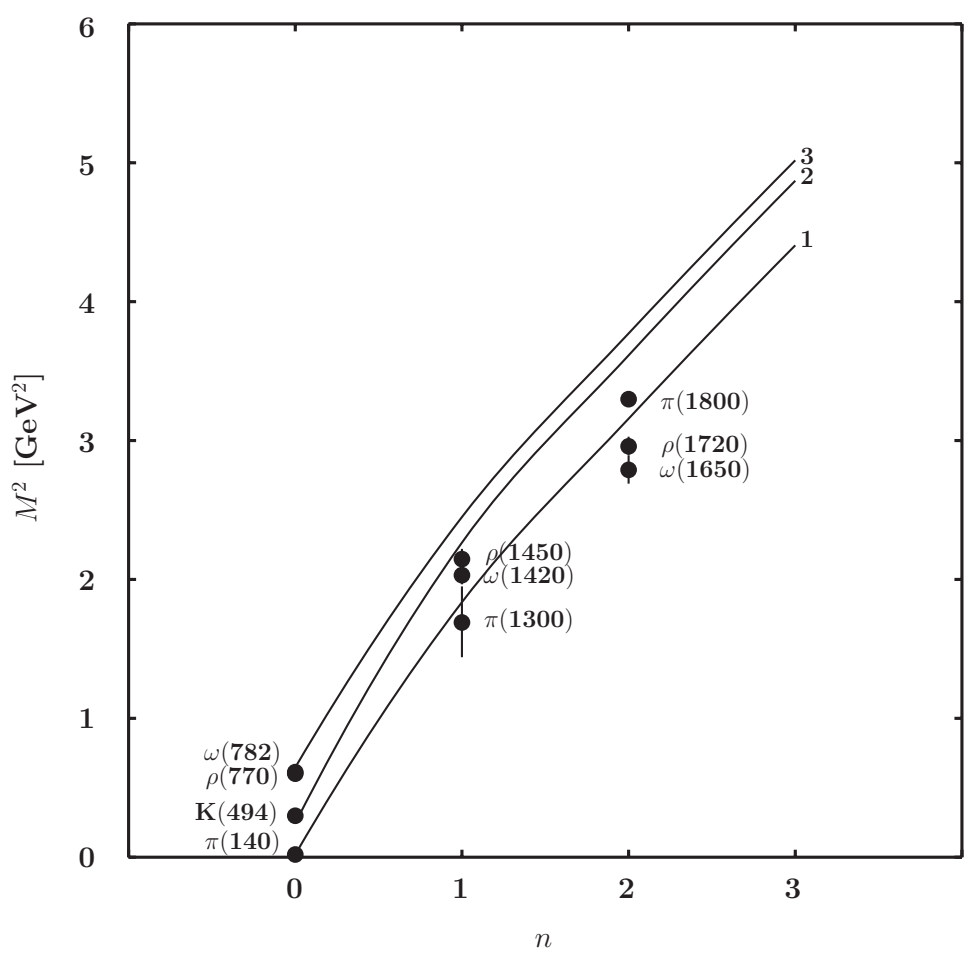

FIG. 2: Mass spectrum of $\pi, K, \rho$ and $\omega$ mesons in dependence on $n$. Central values of data are indicated by black circles (for a few states error bars are included when they are sizeable). The numbers mark the corresponding family trajectories: 1 - $\pi$ mesons; 2 - $K$ mesons; $3-\rho, \omega$ mesons. 


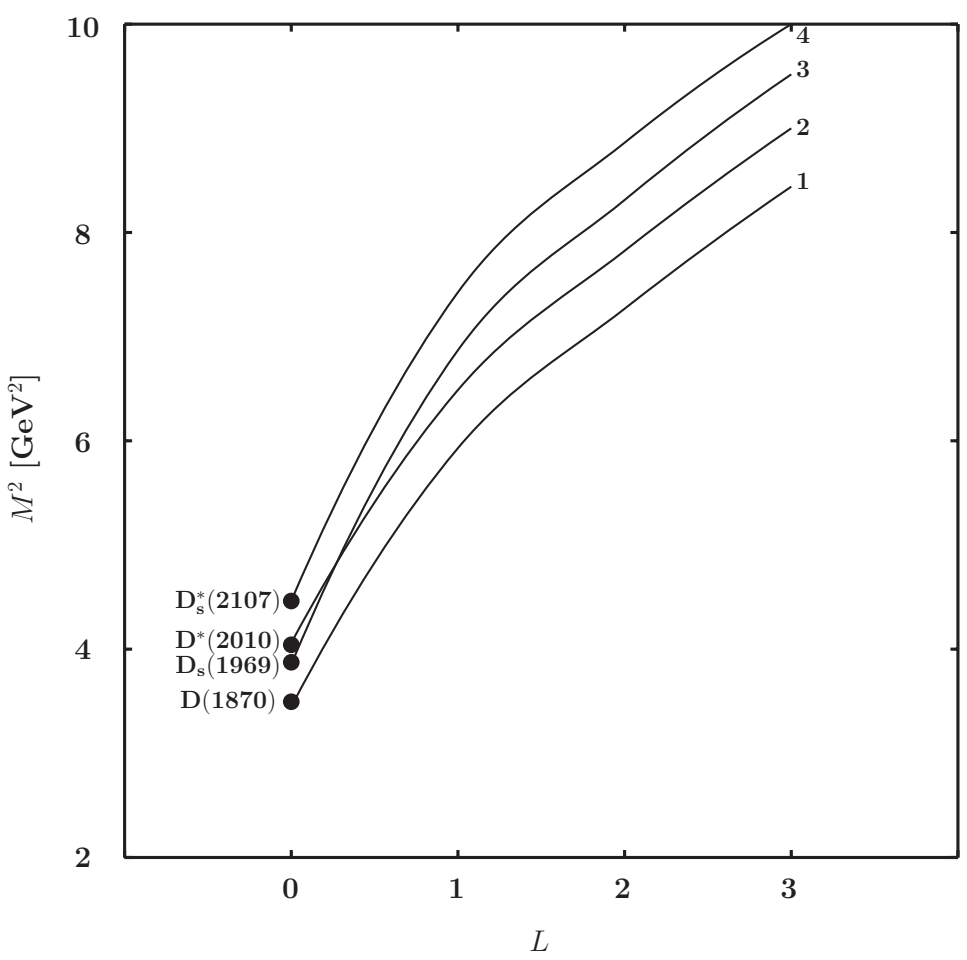

FIG. 3: Mass spectrum of charmed mesons in dependence on $L$. Central values of data are indicated by black circles. The numbers mark the corresponding family trajectories: $1-D$ mesons; $2-D^{*}$ mesons; $3-D_{s}$ mesons; 4 - $D_{s}^{*}$ mesons.

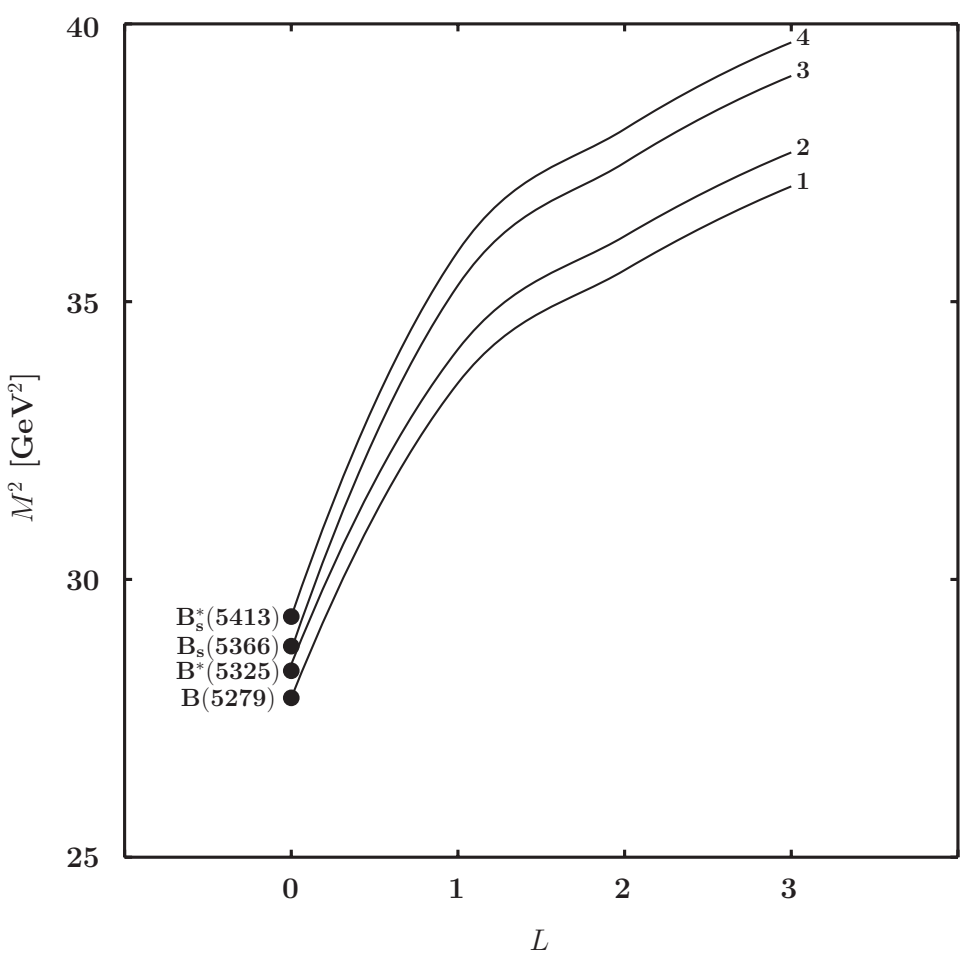

FIG. 4: Mass spectrum of bottom mesons in dependence on $L$. Central values of data are indicated by black circles. The numbers mark the corresponding family trajectories: $1-B$ mesons; 2 - $B^{*}$ mesons; $3-B_{s}$ mesons; 4 - $B_{s}^{*}$ mesons. 


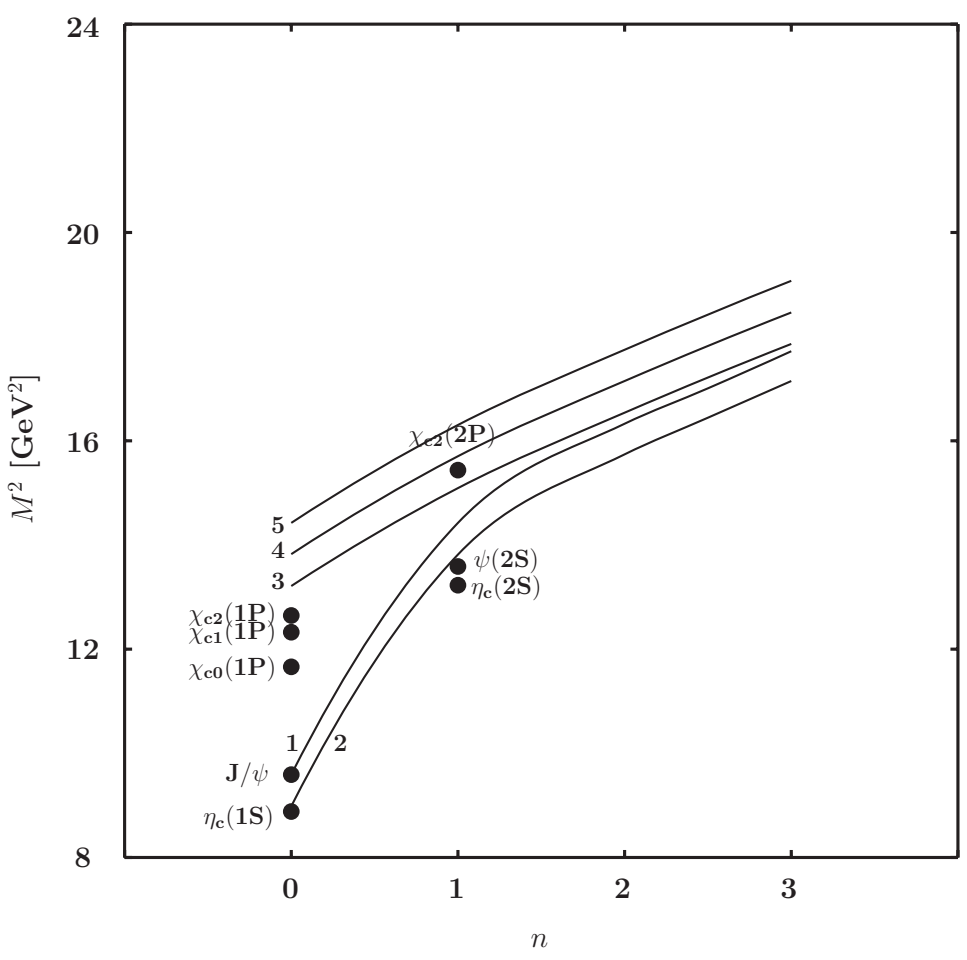

FIG. 5: Mass spectrum of charmonium states. Central values of data are indicated by black circles. The numbers mark the corresponding family trajectories: $1-\psi$ mesons; $2-\eta_{c}$ mesons; $3-\eta_{c 0}$ mesons; 4 - $\eta_{c 1}$ mesons; 5 - $\eta_{c 2}$ mesons.

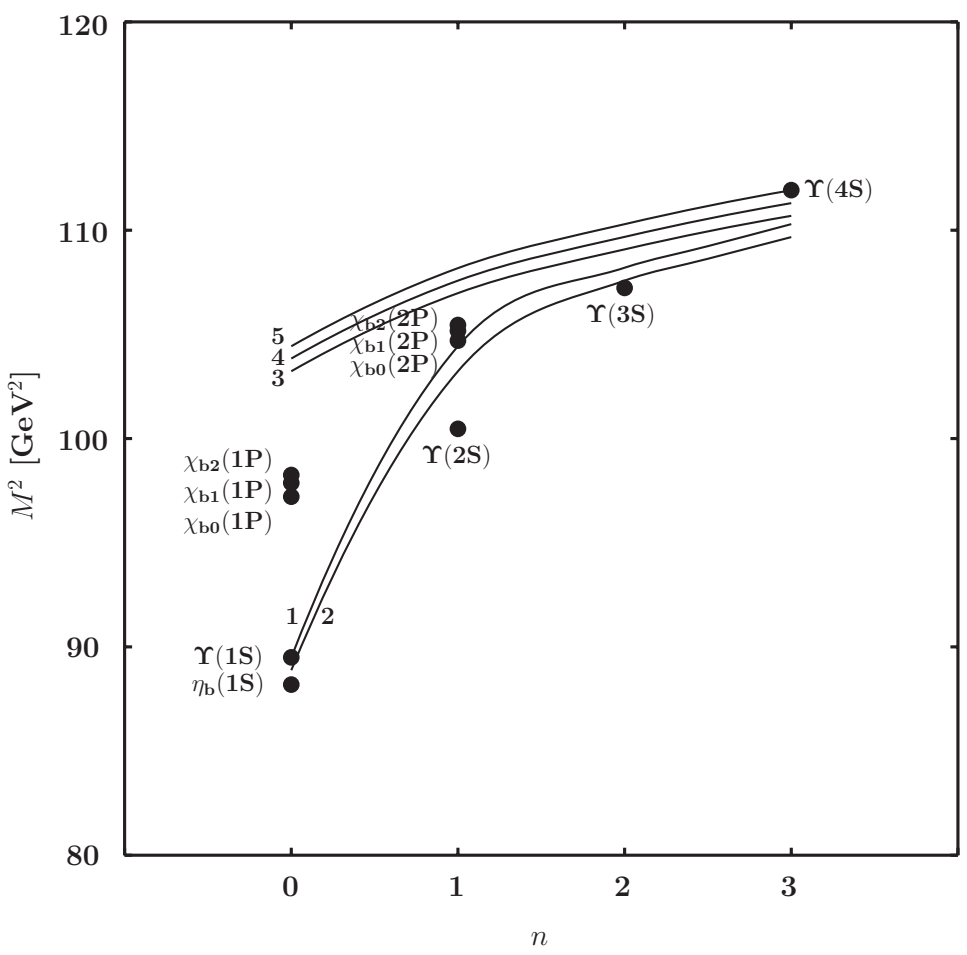

FIG. 6: Mass spectrum of bottomium states. Central values of data are indicated by black circles. The numbers mark the corresponding family trajectories: $1-\Upsilon$ mesons; $2-\eta_{b}$ mesons; $3-\chi_{b 0}$ mesons; $4-\chi_{b 1}$ mesons; 5 - $\chi_{b 2}$ mesons. 